\title{
SATELLITE BEAM HANDOVER STRATEGY TO IMPROVE RETURN LINK RESOURCE UTILIZATION
}

\author{
Jinyoung Jang ${ }^{1}$, Minwoo Lee ${ }^{1}$, Eunkyung Kim ${ }^{1}$ and Jaesung Lim $^{2}$ \\ ${ }^{1}$ Department of Network Centric Warfare, Graduate school of Ajou University, \\ SuWon, South Korea \\ powerblow@nate.com, iminu@ajou.ac.kr, moonnstar@ajou.ac.kr \\ ${ }^{2}$ Jangwee Research Institute for National Defence, Graduate school of Ajou University, \\ SuWon, South Korea \\ jaslimeajou.ac.kr
}

\begin{abstract}
Mobile communication on the multi-beam satellite communication has attracted attention, since Digital Video Broadcasting - Return Channel Satellite (DVB-RCS) has been successively implemented. In general, multi beam satellite employs uniform allocation of RF power and bandwidth to multi beams, since it is hard to predict the distribution of traffic requests and the arrival rate of new connection. This makes it difficult to satisfy the traffic requests in a beam. Thus, when an unpredictable event, such as a disaster, regional warfare and deteriorating weather conditions, occur in a special spot beam, many more terminals will require access to a special beam channel. This causes the special beam channel to be saturated, while adjacent beam channel resources are available. Thus, we propose a novel handover strategy to improve satellite return link resource utilization using load balancing handover. Simulation results shows the proposed handover algorithm outperforms conventional handover algorithms in terms of new connection block probability, handover block probability, return link resource utilization and capacity request block rate.
\end{abstract}

\section{KEY WORDS}

Satellite beam handover, DVB-RCS, return link resource utilization, Load balancing

\section{INTRODUCTION}

Satellite communication system is essential to the maritime and aerospace environment, where infrastructure is not established. Only satellite communication can support broadband network service to maritime terminals, such as merchant ships, naval vessels, maritime platforms and fishing boats.

A global beam satellite system could support a wide range of services. However, the transmission power of a satellite is distributed as widely as the service region. Thus, the power density decreases, according to the service region. Thus multi spot beam satellite is attractive in terms of improving power flux density, system capacity and spectral efficiency[1].

If a terminal moves to another spot beam, a handover will occur between the two beams in the satellite system. In this case, handover detection should be considered. Traditionally, handover detection is based on the RSS (Received Signal Strength) measurement to guarantee QoS (Quality of Service) in the terrestrial cellular communication system. However, there is

DOI : 10.5121/ijwmn.2011.3509 
International Journal of Wireless \& Mobile Networks (IJWMN) Vol. 3, No. 5, October 2011

little difference in RSSs between the centre and the boundary of a satellite beam, whereas a terrestrial communication system has RSS noticeable differences. The satellite RSS can be changed by antenna gain loss and atmospheric attenuation or a jamming signal. A spot beam is divided into high beam area and low beam area due to the rain attenuation margin. The position based handover detection approach is suggested in the DVB-RCS standard as a baseline, since RSS of an adjacent beam is not severely different.

In general, multi beam satellite employs uniform allocation of RF power and bandwidth to multi beams, since it is hard to predict the distribution of traffic requests and arrival rate of new connections. Also, heterogeneous allocation of RF power and bandwidth to multi beams can cause channel interference to adjacent beams. Thus, satellite does not have sufficient flexibility to satisfy the traffic requests on a beam. When an unpredictable event, such as a disaster, regional warfare and deteriorating weather conditions, occur in a special spot beam, it is important to ensure a new connection to the satellite channel[2][3]. However, if many terminals require access to the special beam channel, the speci2l beam channel will be saturated and new connection request will be blocked, while adjacent beam channel resource is available. Even though a new connection request is unpredictable, a handover request can be predicted. Therefore, if the resource utilization of a special beam is exceeds that of the adjacent beam, NCC (Network Control Centre) can recommend terminals that are in the low beam area of the adjacent beam to handover to the adjacent beam, to prevent saturation of the special beam resource. Thus, we propose a load balancing handover strategy that increases satellite return link resource utilization.

The remainder of this paper is organized as follows. Section 2 describes the background of the DVB-RCS and related work on the handover detection algorithm. Section 3 describes characteristics of multi-beam satellite signals. Section 4 presents the proposed load balancing handover strategy. Section 5 presents simulation results. Finally, we conclude this paper in Section 6 .

\section{BACKGROUND AND RELATED WORK}

\subsection{DVB-RCS handover strategy}

The DVB-RCS standard is set up to support mobility of RCST, and proposed the process of beam handover. The handover strategy is processed in three phases, as follows. First phase: Handover detection determines the necessity for the mobile RCST to be handed over, and typically also determines a list of candidate beams. The position based handover detection approach is suggested in the standard as a baseline. The handover detection process can be distributed by RCST or centralized by NCC. Second phase: the handover decision phase selecting the target beam to be handed over, considering the candidate beam resource, and issues handover command. Third phase: the handover execution hands over RCST from a set of resources in the current beam to another set of resources allocated in the target beam [4].

Position based spot beam handover is suited to the DVB-RCS system, because position information is already a requisite in mobile DVB-RCS. RCST uses the SAC (satellite access control) field in SYNC slot to request capacity, send CSI (channel state information) and mobility control messages to NCC. RCSTs periodically send a SYNC burst every super frame [5][6]. A RCST monitors its own position or RSS (received signal strength) to detect the handover necessity. The RCST sends a handover request message to the NCC when handover is detected. This is the distributed handover detection approach. Otherwise, a centralized handover detection approach, in which all processing is done in the NCC, is used. Then, NCC makes the handover decision. Handover execution is initialized after the handover decision 
processes. NCC sends the handover command to the RCST with information about the target beam to be used. Fig. 1 shows the overall DVB-RCS beam handover process.

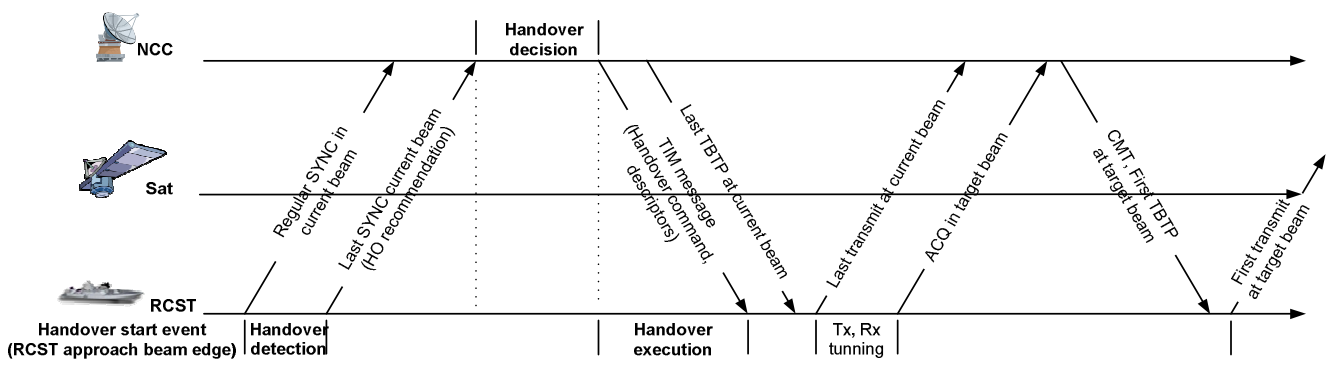

Fig. 1. DVB-RCS handover process

\subsection{Handover detection algorithm}

Traditional handover detection algorithms are based on the RSS measurement. Hysteresis margin and RSS threshold are used to prevent the ping-pong effect. The authors in [7] proposed the adaptive handover algorithm for a multi-beam GEO satellite. They proposed a sampling algorithm and adaptive handover algorithm. The amount of RSS sampling is determined by the velocity of a terminal in the RSS sampling algorithm. The RSS hysteresis margin is determined adaptively by sampling measurement and velocity of a terminal in the adaptive handover algorithm. This algorithm shows that the hysteresis margin decreases when RSS measurements decrease and the terminal velocity is faster. The authors in [8] proposed the adaptive handover algorithm based on distance information. The RSS hysteresis margin is determined adaptively by the distance between the MS (Mobile Station) and the serving BS (Base Station). This algorithm shows that the MS position is close to the cell radius, the hysteresis margin decreases, so as to easily handover trigger. If the distance between the MS and the serving BS is equal to the cell radius, the hysteresis margin becomes zero. The authors in [9] proposed the adaptive handover algorithm based on RCST mobility information. This algorithm shows the better handover performance in the rain sky and shows the better forward link resource utilization.

\subsection{Handover decision / execution algorithm}

The authors in [10][11] proposed DVB-RCS handover scheme for supporting handover decision. After handover detection, if target beam resources are enough to support to handover, handovers immediately decided. However, if not, handover request may be queued. Handover decisions that rely on exclusively on position information without any prioritization strategy may lead to high handover failure rates. So they classified of a RCST such as aircraft, highspeed train by the velocity. RCSTs estimate of residual time of current spot beam by using of a target beam approach velocity (TBAV) and a distance of beam edge. handover request is prioritized by the NCC according to the residence time value. This handover decision algorithm shows that lower handover failure probability and higher new connection blocking probability. The authors in [12] proposed handover execution algorithm. They pay attention to the process of after handover decision. After handover decision, NCC sends a TIM (terminal information table) message to the RCST. If TIM message is lost, handover execution will be fail. So they proposed the NCC with memory for the lower the handover failure probability. Briefly, the prior researches only pay attention to the after position based handover detection 
International Journal of Wireless \& Mobile Networks (IJWMN) Vol. 3, No. 5, October 2011

phase. On the other hand, we concentrate on the phase of handover detection to increase return link resource utilization.

\section{CHARACTERISTICS OF THE MULTI-BEAM SATELLITE SIGNAL}

\subsection{Characteristics of terrestrial signal}

Generally, handover detection is based on RSS in terrestrial communication systems. RSS is affected by small fading and large fading in the case of the terrestrial system. Small fading is caused by delay spread and the Doppler Effect. Large fading is caused by shadow fading. Thus, terrestrial RSS is can be expressed as Eq. (1) [13].

$$
\mathrm{P}_{\mathrm{R}}=\mathrm{P}_{\mathrm{T}} \cdot \mathrm{G}_{\mathrm{R}} \cdot \mathrm{L}^{-\mathrm{a}} \cdot 10^{\frac{\theta}{10}}
$$

Where, $\mathrm{P}_{\mathrm{R}}, \mathrm{P}_{\mathrm{T}}, \mathrm{G}_{\mathrm{R}}, \mathrm{L}$, -a and $\theta$ represent RSS, transmitter power, antenna gain, distance between transmitter and receiver, path loss exponent, and shadow fading component, respectively. However, rapid fluctuations due to small fading can be ignored by being averaged over sampling. Thus, the main reasons of variation of RSS are shadowing and path loss. Thus, RSS is highly dependent on location and the time condition of a terminal. Therefore, the RSS of a terrestrial system is largely changed. Therefore, if the terminal is handed over to another cell, the RSS hysteresis margin must be sufficiently large to prevent a ping pong effect.

\subsection{Characteristics of multi-beam satellite signal}

Satellite RSS can be expressed as Eq. (2). [14].

$$
P_{R}=\frac{P_{T} \cdot G_{T} \cdot G_{R}}{L_{P} \cdot L_{T X} \cdot L_{A}}
$$

where, $P_{R}, P_{T}, G_{T}, G_{R}, L_{P}, L_{T X}, L_{A}$ represent RSS, transmitter power, transmitter antenna gain, receiver antenna gain, path loss, transmitter antenna gain loss of position offset, and atmospheric attenuation, respectively. There is very little difference of the distance between GEO satellite and center and edge of the beam, since the satellite system in maritime and aerial environments is LOS (Line Of Sight). Thus, there is very little difference of path loss. Therefore, the RSS of a satellite system is not change greatly. The main reason of variation is antenna gain loss of the position offset and atmospheric attenuation.

In general, the satellite directional antenna gain loss of position offset is $3 \mathrm{~dB}$ at the beam boundary. RSS can be more attenuated by interference of an adjacent beam at the beam boundary. Therefore, antenna gain loss of the position offset must be considered, when designing the link budget of a satellite system. The antenna gain loss of position offset can be expressed as Eq. (3). [4]

$$
\mathrm{L}_{\mathrm{TX}}=12 \cdot\left(\frac{\theta_{\mathrm{T}}}{\theta_{3 \mathrm{~dB}}}\right)^{2}
$$

Where, $\mathrm{L}_{\mathrm{TX}}, \theta_{\mathrm{T}}$, and $\theta_{3 \mathrm{~dB}}$ represent the transmitter antenna gain loss of position offset, angle of satellite to RCST, and beam width, respectively. Fig. 2 shows the antenna gain loss of position offset according to its relative off axis angle, with a beam width of $1^{\circ}$ 


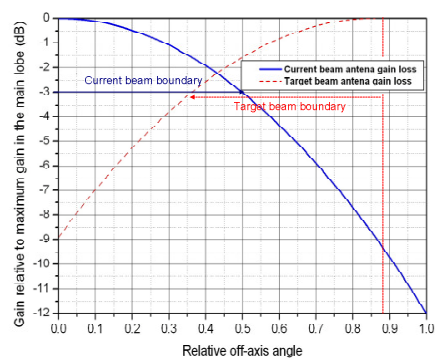

Fig. 2. Antenna gain loss of position offset

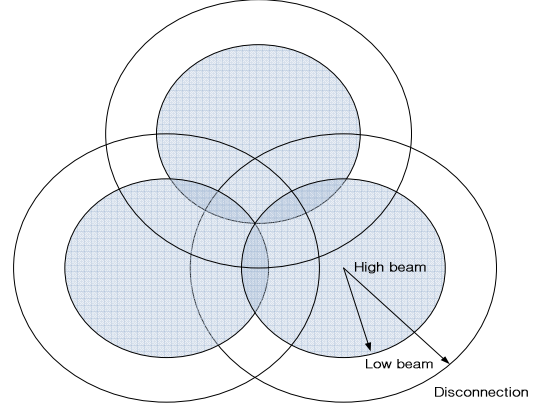

Fig. 3. Spot beam arrangement

The second variation factor is Atmospheric attenuation. This atmospheric attenuation is highly time dependent. Generally, atmospheric attenuation is very small (e.g. 0.2dB). However, when it is raining, satellite RSS is severely influenced by rain attenuation at the ku, ka band (e.g. $5 \mathrm{~dB}$ ). Thus, a satellite system is designed to overcome rain attenuation. Accordingly, the spot beam is divided into a high beam area and a low beam area, as in Fig.3.

If CCM (Constant Coding and Modulation) is applied to a satellite system in clear conditions, satellite communication is guaranteed in the low beam area caused by the rain attenuation margin. DVB-S2 has recently applied the ACM (Adaptive Coding and Modulation) to increase resource utilization. Thus, even if handover detection is delayed in the low beam area, the QoS of satellite communication could be guaranteed. However, the overall spectral efficiency will be degraded.

\section{LOAD BALANCING HANDOVER STRATEGY}

The special spot beam channel will be saturated, if there are many unexpected new connection requests to the special spot beam channel, while the adjacent beam channel resource is available.

This blocks the new connection request and the handover request to the special spot beam. However, a mobile terminal that is in the region of adjacent low beam boundary could be guaranteed QoS, even if handed over to the adjacent beam. Thus, we can prevent saturation of special spot beam resource by handing over mobile terminal to the adjacent beam when saturation of the special spot beam resource occurs, while adjacent beam resource is available and the mobile terminal is in the low beam boundary of adjacent beam. That is, we can balance loads by handing over the mobile terminal. Thus, we proposed the load balancing handover strategy.

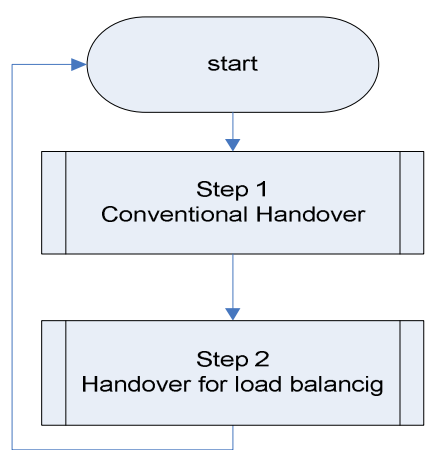

Fig. 4. Proposed handover strategy 
Fig. 4 shows the proposed overall handover procedure. First step: NCC or RCST recommends handover to the adjacent beam by the conventional handover detection algorithm. Next step: NCC compares the resource of the current beam and target beam, and if it expects saturation of the special beam channel, hands over RCST to the adjacent beam to balance the load. The detail of steps 1 and 2 will be described in sub section 4.1 and 4.2 , respectively.

If we use the step 2 handover algorithm, terminals in the low beam boundary of the adjacent beam can be handed over. However, Conditions (RSS or position) of the current beam are better than that of the adjacent beam.

Thus, if the RSS based handover detection algorithm, such as SHM (Static Hysteresis Margin), AHM (Adaptive Hysteresis Margin), or AHM (Adaptive Hysteresis Margin, based on distance information) is used as the step 1 conventional handover detection algorithm, oscillating handover occurs. Thus, we proposed the RCST mobility based handover detection algorithm with ADM (Adaptive Distance Margin) for step 1 [9]. the load balancing handover algorithm, using mobility information of RCST as a step 2, to prevent oscillating handover.

\subsection{Step 1: handover detection algorithm based on RCST mobility information}

Fig.4 describes the overall handover detection situation.

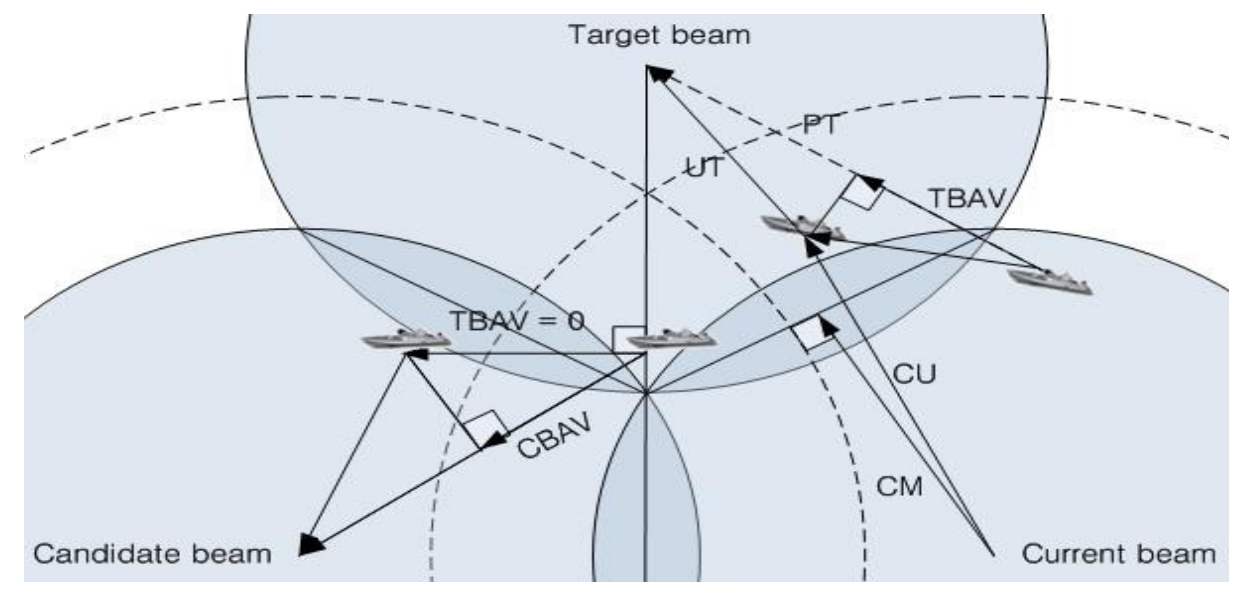

Fig. 5. Proposed handover detection situation

The target beam approach velocity of RCST is calculated as in Eq. (4)

$$
\mathrm{TBAV}_{\mathrm{m}}=\max \left(|\mathbf{P T}|-\mid \operatorname{proj}_{\mathbf{P T}}(\text { UT }) \mid, \quad 0\right)
$$

Where, $\mathbf{T B A V}_{\mathbf{m}}, \mathbf{U T}$ and $\mathbf{P T}$ represent target beam approach velocity of RCST per minute, the vector that current position of the RCST to the center of target beam and the vector that preposition of the RCST to the center of target beam, respectively. The adaptive distance margin is calculated as in Eq. (5)

$$
\mathrm{d}_{\mathrm{Am}}=\frac{8}{\sqrt{\text { TBAV }}} \times\left(\frac{|\mathbf{C U}|}{|\mathbf{C M}|}\right)^{5}
$$


The distance margin adaptively adjusted by position of the RCST and TBAV using Eq. (5). Where $\mathrm{d}_{\mathrm{Am}}$, and $\mathbf{C U}, \mathbf{C M}$ represent adaptive distance margin, the vector that is the center of the current beam to the current position of the RCST, and the vector that is the center of the current beam to the middle point between the current beam and the target beam center.

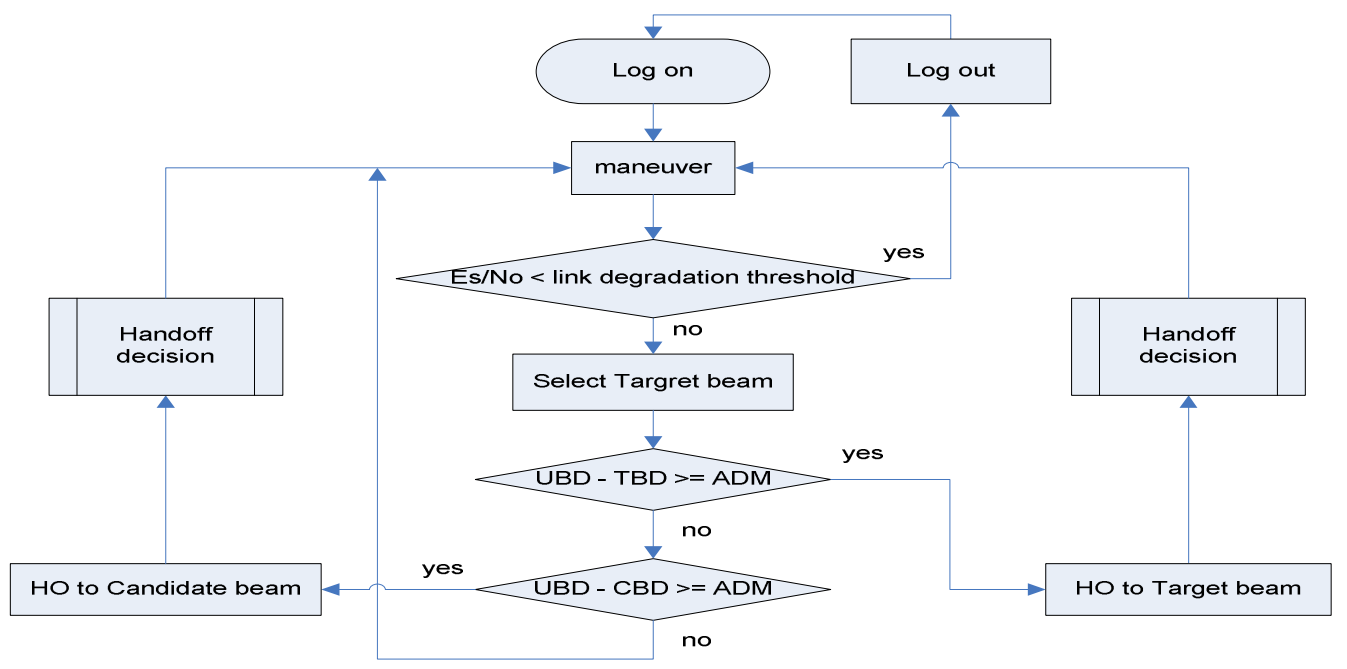

Fig. 6. Overall handover detection procedure

Fig.6. shows that the overall handover detection procedure. The Log on RCST measures the channel state. If the channel state is lower than the link degradation threshold, RCST may log out, or RCST ranks the adjacent beams in order of the closest distance from the beam center. Then, it selects the first ranked adjacent beam as a target beam, and the next ranked beam as a candidate beam. Next, RCST or NCC performs the Adaptive handover detection algorithm based on RCST mobility information. If the current beam distance (UBD) is greater than the sum of the target beam distance (TBD) and the adaptive distance margin (ADM), RCST is handed over to the target beam. However, if the RCST is not in the current high beam boundary but in the target high beam boundary, and RCST is moving away from the target beam center, as a Fig.5. Then, handover detection is delayed until the target beam is changed. This increases the link degradation rate or lowers resource utilization. Thus, it is desirable that it considers candidate beam handover. If the UBD is greater than the sum of candidate beam distance (CBD) and ADM, RCST is handed over to the candidate beam.

\subsection{Step 2: Handover algorithm for load balancing}

It is hard to predict the distribution of the traffic request and the new connection arrival. Conversely, the handover request of terminals can be predicted. The RCST that is closer and approaches the target beam faster is more likely to be handed over to the target beam.

It is desirable that handover RCST, which is the most expected handover to the adjacent beam, in the process of step 2. This means that terminals are handed over in advance to balance loads. Thus, we proposed a LDM (Load balancing Distance Margin) concept to select the anticipated handover terminal. 
International Journal of Wireless \& Mobile Networks (IJWMN) Vol. 3, No. 5, October 2011

$$
\mathrm{LDM}=\frac{1}{\sqrt{\mathrm{TBAV}_{\mathrm{m}}}} \times \frac{|\mathbf{U T}|^{2}}{|\mathbf{C U}|}
$$

As the RCST is closer and approaches to the target beam faster, LDM is going to be smaller to be easily handed over to the target beam. The LDM of RCST is calculated as in Eq. (6)

$$
\mathrm{LBD}=\tan \left(\sqrt{(3+\text { Rain margin }) / 12} \times \frac{\theta_{3 d B}}{180 \pi}\right) \times 36000
$$

The LBD (Low Beam boundary distance) is calculated as in Eq. (7) from Eq. (3). If RCSTs are in the LBD of the adjacent beam under a clear sky, RCST can be serviced without link degradation.

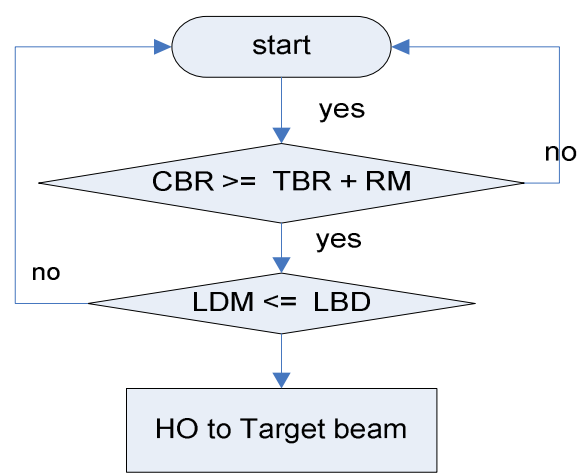

Fig. 7. Overall handover for the load balancing procedure

Fig. 7. shows that the overall handover process for the load balancing procedure. If CBR (Current Beam Resource) is greater than the sum of TBR (Target Beam Resource) and RM (Resource Margin), NCC tries to balance loads. If the LDM of terminal is smaller than LBD, RCST is handed over to the target beam. RM is necessary to prevent the back and forth handover caused by load balancing handover.

Load balancing of multi beams is achieved by handing over the anticipated handover RCST in advance. Furthermore, oscillating handover does not occur, because it is considered by TBAV in the step1 and step 2 algorithms.

\section{SiMULATION RESULTS}

This section evaluates performances of the proposed handover strategy in terms of new connection block probability, handover block probability, return link resource utilization, handover rate and link degradation rate. Simulation is done based on matlab ver 7.0.4. Simulations show the proposed load balancing handover strategy achieved better performance than conventional handover strategy did. Table 1 lists the simulation parameters. 
International Journal of Wireless \& Mobile Networks (IJWMN) Vol. 3, No. 5, October 2011

Table 1 Simulation parameters

\begin{tabular}{|c|c|}
\hline parameters & value \\
\hline Simulation time & 10 hour \\
\hline Number of beams & 3 \\
\hline Beam width & $0.5^{\circ}$ \\
\hline Fixed Capacity Allocation per beam & 128 slot \\
\hline Capacity request per RCST & $1 \sim 5$ slot \\
\hline Resource margin & 12 slot \\
\hline Initially number of RCST & 90 \\
\hline Velocity of RCST & $6 \sim 1000 \mathrm{kmph}$ \\
\hline Course change rate of RCST & $5^{\circ} \sim 15^{\circ} / 15 \mathrm{~min}$ \\
\hline Average service time & $5 \mathrm{hour}$ \\
\hline Threshold of link degradation & $-126.8085 \mathrm{~dB}$ \\
\hline Rain attenuation margin & $5 \mathrm{~dB}$ \\
\hline
\end{tabular}

We assumed that the FCA (Fixed Capacity Allocation) per beam is 128 slots. The new connection arrival process in each beam is Poisson with rate $\lambda$. The average service time is exponentially distributed with a mean of 5 hours. The load balancing handover resource margin is 12 slots, 90 RCST is initially distributed uniformly in each beam. We concentrate on the effectiveness of load balancing handover. Performance is compared to the conventional handover algorithm without load balancing handover and the proposed handover strategy using LDM. Conventional handover algorithms achieved similar performance from the viewpoint of resource utilization. Whereas, proposed handover strategy achieved better performance. The proposed strategy not only serviced more RCSTs, but also achieved better QoS per RCST.

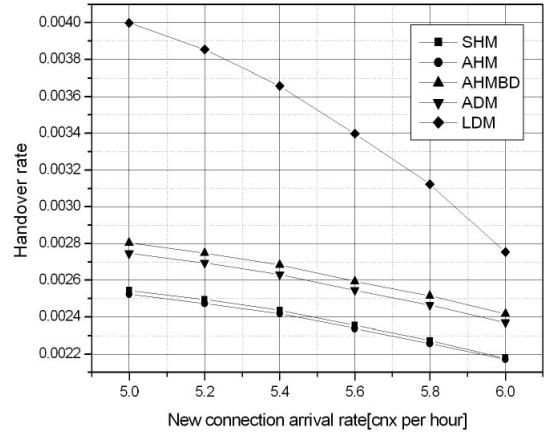

Fig. 8. Handover rate vs. $\lambda$

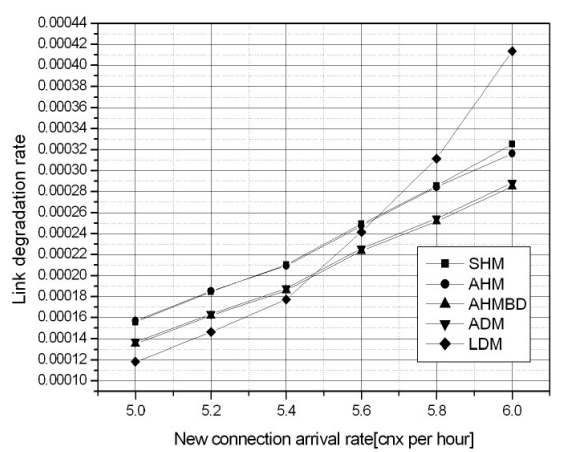

Fig. 9. Link degradation rate vs. $\lambda$

Fig. 8 shows the handover rate versus the new connection arrival rate[ $\lambda]$. Handover rate decreases with increasing $\lambda$, as the available resources of a spot beam decrease with increases in the new connection arrival. The handover rate of the proposed algorithm, LDM, is greater than that of conventional handover algorithms that do not apply a load balancing strategy Due to the step 2 handover procedure of the LDM scheme to balance loads after the conventional handover procedure. Therefore, the proposed handover strategy has a disadvantage from the viewpoint of the handover rate. 
However, the Return link resource utilization, service rate and QoS per RCST are greater. Fig.9. shows the link degradation rate according to the new connection arrival rate $[\lambda]$. As $\lambda$ increased, the link degradation rate increased. LDM shows the lowest link degradation rate until the new connection rate was 5.6. If the channel condition of the terminal is out of the link degradation threshold, the terminal is logged out. Therefore, terminals must be handed over to the target beam before link degradation occurs. Especially, a fast terminal has a short time until the link degradation worsens. If the handover request is blocked due to the shortage of target beam resource, and the handover does not succeed until the link degradation worsens, the terminal is logged out. The lowest link degradation rate is achieved at the LDM using target beam approach velocity in step 1 and step 2 .

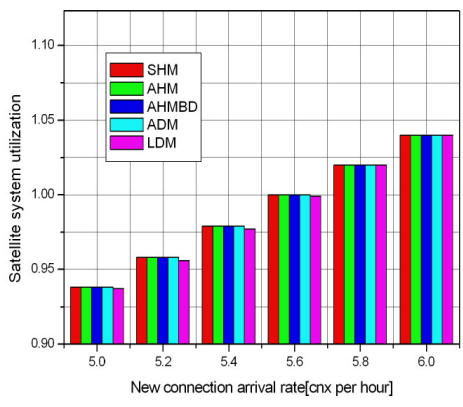

Fig. 10. Satellite system utilization vs. $\lambda$

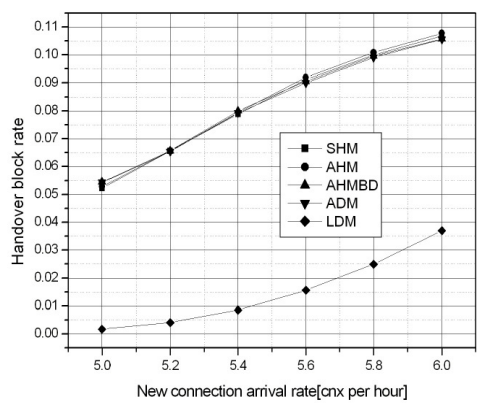

Fig. 11. Handover block rate vs. $\lambda$

Fig.10 shows the satellite system utilization $[\rho=\lambda / \mu]$ based on the new connection arrival rate. A new connection arrival rate above 5.6 overflows the satellite system. So plots higher than $\lambda=5.6$ are meaningless. This result shows that the proposed handover strategy achieved a better service rate $[\mu]$. That is, more RCSTs were serviced by the satellite when we applied LDM. Fig.11 and Fig.12 show the handover block rate and new connection block rate, respectively. LDM achieves that the lowest handover rate and new connection rate, according to the new connection arrival rate. Other schemes show similar performance. They do not apply the step 2 load balancing handover. Thus, the special spot beam channel can be saturated, while the adjacent spot beam channel is available. Whereas, saturation of the special spot beam channel is prevented by step 2 load balancing handover at the LDM.

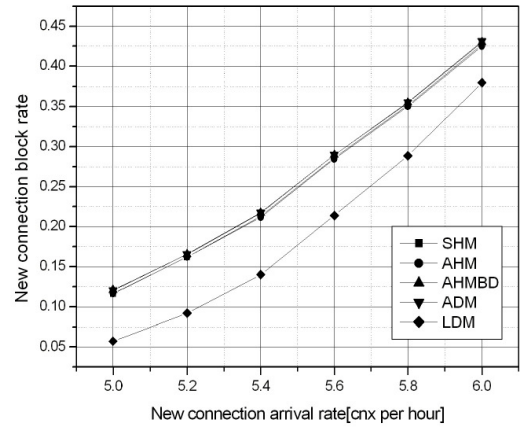

Fig. 12. New connection block rate vs. $\lambda$

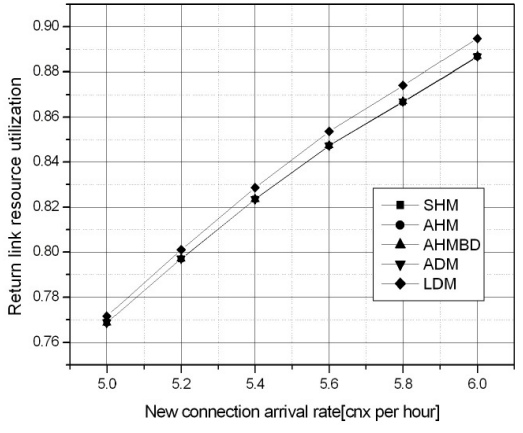

Fig. 13. Return link resource utilization vs. $\lambda$ 
Fig.13 shows the return link resource utilization according to the new connection arrival rate. RCSTs request the resource used in the return link, by transmitting the CR (Capacity request) message within the SAC field. NCC allocates the return link capacity to the RCST. Extra resource is allocated to RCSTs by FCA (Free Capacity assignment) strategy, if the return link resource remains after allocation of the CR. In the opposite case, the CR of RCSTs is blocked. This lowers QoS. As the new connection arrival rate increases, total return link utilization increases. LDM shows the highest return link resource utilization. This means that the remainder of the resource is lower after capacity allocation. This result is caused by the low new connection block rate and link degradation rate

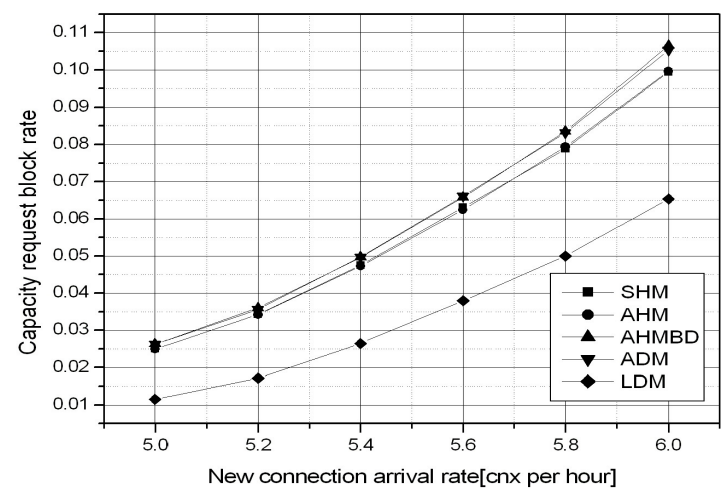

Fig. 14. Capacity request block rate vs. $\lambda$

Fig.14 shows the capacity request block rate versus the new connection arrival rate. As the new connection arrival rate increases, the capacity request block rate increases. LDM achieves the lowest capacity block rate. This means that CR is more allocated at the LDM than other schemes. This result shows that the QoS of a RCST is further guaranteed by step 2 load balancing handover.

\section{CONCLuSion}

The DVB-RCS standard is evolving and was released to support terminal mobility. Mobility and resource management are key issues of a satellite system. NCC manages the resource of each beam and can know mobility information of all RCSTs. If the distribution of traffic and RCST at each beam differs, that is, if the resource utilization of each beam differs, it is possible to achieve the load balancing effect by RCST handover in advance, which is expected to handover to the adjacent beam. Thus, we proposed the spot beam handover strategy to increase return link resource utilization.

The RSS based conventional handover scheme causes oscillating handover, if we apply it to load balancing handover. Therefore, we consider TBAV for the conventional and load balancing handover algorithm to prevent a ping pong effect.

Simulations show low link degradation and a new connection block rate are achieved using our proposed algorithm. This increases return link resource utilization. Furthermore, a low capacity request block rate is achieved. Thus, more RCSTs can be serviced and the QoS of a RCST is increased by load balancing handover. 
International Journal of Wireless \& Mobile Networks (IJWMN) Vol. 3, No. 5, October 2011

\section{ACKNOWLEDGMENT}

This research was supported by the MKE (The Ministry of Knowledge Economy), Korea, under the ITRC (Information Technology Research Center) support program supervised by the NIPA (National IT Industry Promotion Agency" (NIPA-2011-(C1090-1121-0011))

\section{REFERENCES}

[1] Ray E. Sheriff and Y. Fun Hu : Mobile Satellite Communication Networks

[2] X. Alberti, J.M. Cebrian : System Capacity Optimization in Time and Frequency for Multibeam Multi-Media Satellite Systems, ASMS-SPSC 2010, 226-233

[3] Katsuya Nakahira, Kiyoshi Kobayashi and Masazumi Ueba, Capacity and Quality Enhancement using an Adaptive Resource Allocation for Multi-beam Mobile Satellite Communication, WCNC 2006, 153-158

[4] ETSI TR 102768 V1.1.1 (2009-04), Digital Video Broadcasting (DVB); Interaction channel for Satellite Distribution Systems ; Guideline for the use of En 301790 in mobile scenarios

[5] ETSI EN 301768 V1.5.1 (2009-05), Digital Video Broadcasting (DVB) ; Intersection channel for Satellite Distribution Systems

[6] A. Iuoras and C. Morlet : Network and Mobility Management for mobile DVB-S2/DVB-RCS systems, IWSSC 2007, 264-268.

[7] F Li Song, Ai-jun Liu and Yi-fei Ma : Adaptive handover algorithm for multi-beam GEO mobile satellite system, ICC 2008, 1947-1951,

[8] Huamin Zhu and Kyung-sup Kwak : Performance analysis of an adaptive handover algorithm based on distance information, computer communications 30(2007), 1278-1288,

[9] Jinyong Jang, Minwoo Lee and Eunkyung Kim : Satellite beam handover detection algorithm based on RCST mobility information, WASET 2011,779-785

[10] Fabio Lattanzi, Guray Acar and Barry Evans : DVB-RCS spotbeam handover using residence time estimation in vehicular satellite network, $4^{\text {th }}$ ASMS 2008, 235-239.

[11] Fabio Lattanzi, Guray Acar and Barry Evans : Performance study of a lightweight DVB-RCS handover scheme for vehicular GEO Networks, IWSSC 2008

[12] Guray Acar, Paris Skoutaridis, Christos Kasparis and Barry Evans : position-based DVB-RCS spotbeam handover in vehicular Geostationary satellite networks, VTC spring 2007, 1410-1414

[13] Tae Chul Hong, Kun Seok Kang, Do-Seob Ahn and Ho-Jin Lee : Inter- System Handover Analysis in Integrated Terrestrial and GEO satellite Communication Networks for Seamless Mobility, 9th ACT 2007, 717-721

[14] Anil K. Maini and Varsha Agrawal : satellite Technology principles and applications 2007

[15] Syed S.Rizvi, Aasia Riasat and Khaled M. Elleithy : A Quantitative Analysis of Handover Time at MAC Layer for Wireless Mobile Networks, IJWMN 2009,vol 2,no.1, 21-28 